\title{
Measurement of Pulse rate and SPo2 using Pulse Oximeter developed using LabVIEW
}

\author{
T.Bheema lingaiah ${ }^{1}$, D.Hanumesh Kumar ${ }^{2}$, C.Nagaraja ${ }^{2}$ \\ 1. Dept. of Biomedical Engineering, Jimma Institute of Technology, Jimma University, Jimma, ETHIOPIA, \\ 2. Dept of Instrumentation, Sri Krishnadevaraya University, Anantapur, Andhra Pradesh, India.
}

\begin{abstract}
The objective of this study is to develop a pulse Oximeter for the determination of pulse rate and percentage of oxygen in the blood of a patient. To determine these parameters the light is transmitted through the fingertip using a photodiode as a sensor and two LED'S (RED \& IR) as a light source. The photo diode detects the light and the DAQ device outputs a voltage corresponding to the amount of light detected, and the final signal is a pulse. To determine the pulse rate, first the time that elapses between two successive peaks must be determined. Second to calculate the percentage of oxygen, $A C \& D C$ voltages must be determined. Based on voltages the modulation ratio is calculated, which is the ratio of magnitude of RED waveform to that of IR waveform.
\end{abstract}

Keywords: DAQ; sensor; signal processing;

\section{INTRODUCTION}

Pulse oximetery has been one of the most significant technological advances in clinical monitoring in the last two decades. Pulse oximetery is a non-invasive photometric technique that provides information about the arterial blood oxygen saturation $(\mathrm{SpO} 2)$ and heart rate, and has widespread clinical applications. The use of pulse oximeters has been described in many settings: hospital, outpatient, domiciliary use and in veterinary clinics ${ }^{[6]}$.

Pulse oximeters determine arterial blood oxygen saturation by measuring the light absorbance of tissue at two different wavelengths and using the arterial blood pulsation to differentiate between absorbance of arterial blood and other absorbers. A good choice of wavelength is where there are large differences in the extinction coefficients of deoxyhaemoglobin and oxyhaemoglobin. Another criterion for the wavelength selection is the relative flatness of the absorption spectra around the chosen wavelength. The two conventional wavelengths used in pulse oximetery are $660 \mathrm{~nm}$ (red) and $940 \mathrm{~nm}$ (near infrared) ${ }^{[1,2]}$.

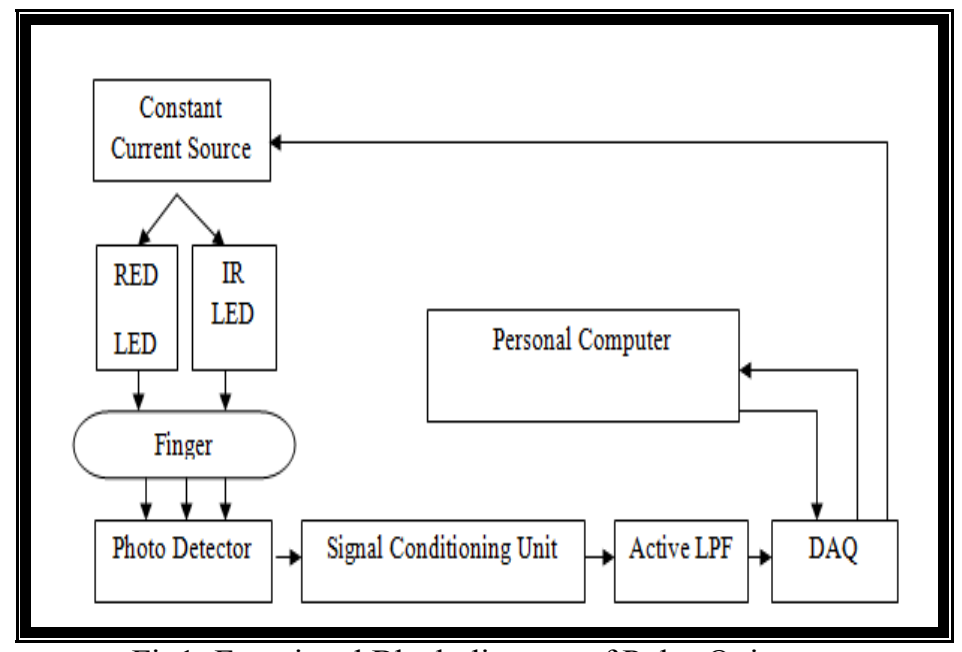

Fig1: Functional Block diagram of Pulse Oximeter

\section{Instrumentation:}

blocks

The block diagram of pulse Oximeter is shown in the fig 1. It consists of the following functional

1. Personal Computer

2. Constant current source

3. Finger probe

4. Signal conditioning unit 
5. Active filter

6. DAQ

The functional approach to the system is described below. The constant current source section consists of transistorized current source for illuminating RED and IR LED's. The photo detector detects the intensity of light transmitted through the finger. The output of the photo detector is amplified and processed by signal conditioning unit. The signal conditioning unit consists of a voltage follower, $50 \mathrm{~Hz}$ noise filter and an amplifier with an adjustable gain. The output of signal conditioning unit is applied to the active low pass filter which eliminates the noises induced by the power supply as well as due to the operations of active components involved in the circuit. The output of the filter is applied to the system for processing through LabVIEW ${ }^{[8]}$ and to display the result through the DAQ.

The switching signal for the constant current source to select RED an IR LEDs will be generated within the system using LabVIEW and sent to the source through the ports of DAQ.

The complete circuit diagram of pulse Oximeter is shown in fig 2.

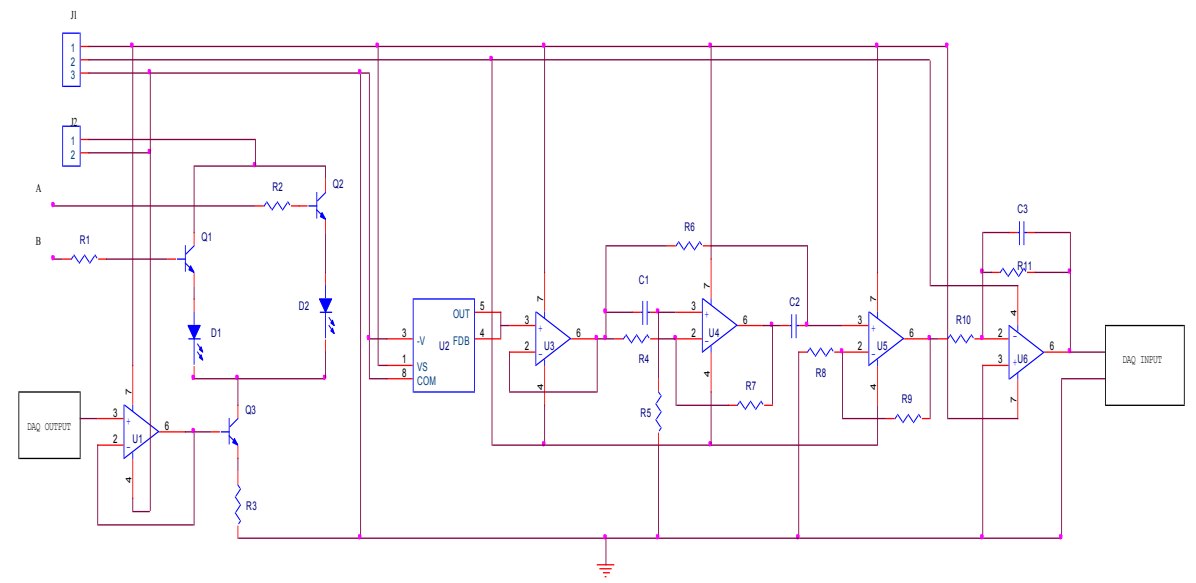

Fig 2: Circuit diagram of Pulse Oximeter

\section{Software Development:}

Lab VIEW program is to calculate:

$>$ Percent oxygenation

$>$ Pulse rate

The percent oxygen ${ }^{[3,5]}$ in the blood of a patient can be calculated by measuring the amount of light that is transmitted through the fingertip using a photodiode as a sensor and two LEDs (red and IR) as the light source. The photodiode detects the transmitted light and the DAQ device outputs a voltage that corresponds to the amount of light detected. The final signal appears as the waveform of a pulse. There will be two waveforms: one signal for the red LED and a second for the IR LED. Once these signals are generated using the DAQ device, several measurements are made. First, the amount of time that elapses in between peaks will be determined in order to calculate the pulse rate. Second, the AC and DC of the voltage signals will be determined in order to calculate the percent of oxygenation. Once these values are found, the modulation ratio ${ }^{[4]}$ is calculated. The modulation ratio is essentially the ratio of the magnitude of the red waveform to that of the IR waveform ${ }^{[9]}$.

To be able to record and calculate all of these measurements, the code is split into a series of events that occur from left to right of our code.

The first event that occurs is turning ON the red LED by generating step voltage using the DAQ ${ }^{[7]}$ device, this is shown in the first frame of the flat sequence structure used to execute the developed code.

Then, the second event occurs which includes several steps:

$>$ The RED LED is switched on.

> The light transmittance is detected by the photodiode and outputs as a voltage signal for 5 secs using DAQ device input.

$>$ The generated signal is cut into a section containing time between 3 secs to 8 seconds

$>$ The DC (average) value between 3 to 8 secs is calculated

$>$ The residual's peak to peak (AC) voltage value is found out.

$>$ The time interval in between peaks is found.

$>$ The peak to peak time interval obtained is divided by 60 in order to obtain the pulse rate. structure.

All the above steps are performed by the sub diagram placed in the second frame of the flat sequence 
The third event turns on the IR LED and turns off the Red LED by generating step voltage using the DAQ device; this is shown in the third frame of the flat sequence structure.

The fourth event includes is a repeat of the second step, however all values will be found for the IR signal instead of the red LED signal. Also, it is unnecessary to find the peak to peak time interval for the IR signal because the pulse rate should be the same for both LED signals, and therefore, calculating pulse rate from the red LED only is sufficient. This is performed by the sub diagram placed in the fourth frame of the flat sequence structure.

The fifth event turns off both Red and IR LEDs.

After these values are determined, the modulation ratio can be calculated by using the equation $\mathrm{R}=(\mathrm{AC} / \mathrm{DC})$ $\mathrm{RED} /(\mathrm{AC} / \mathrm{DC}) \mathrm{IR}$. Then, the empirical data is referenced in order to find the percentage of oxygenation.

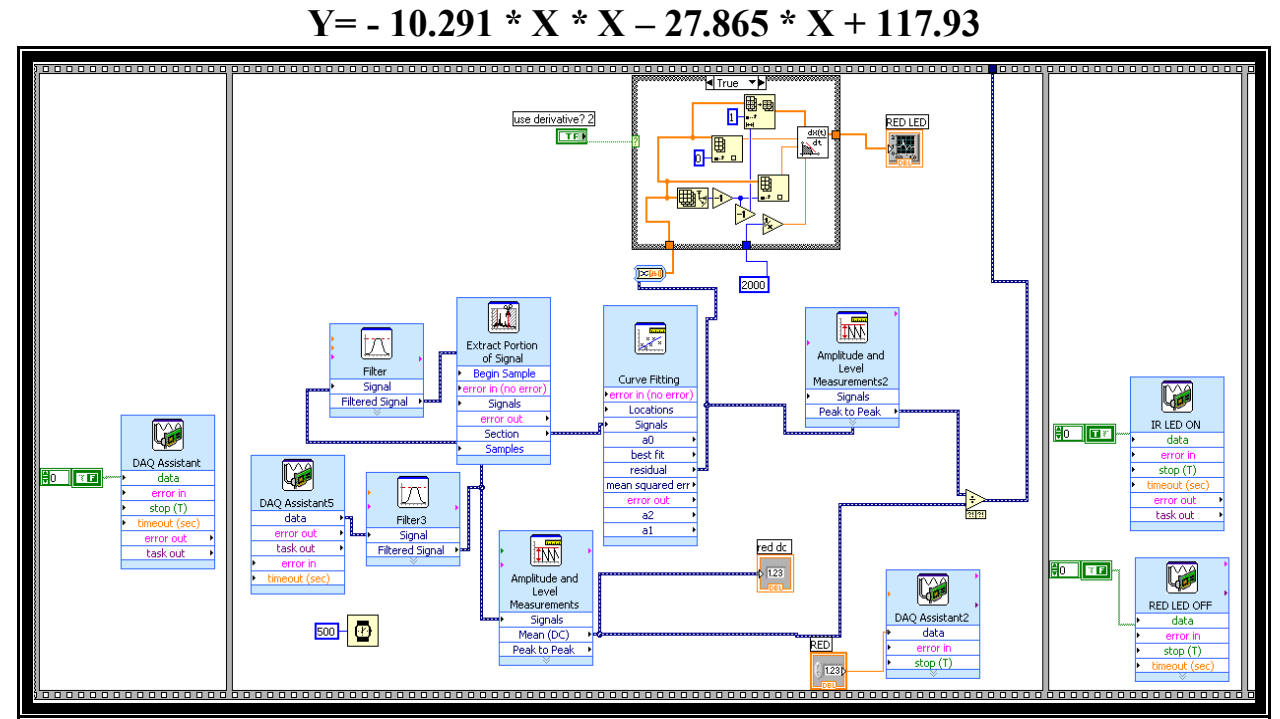

Fig 3(a): VI Block diagram of Pulse Oximeter for Turning on RED LED ON and OFF

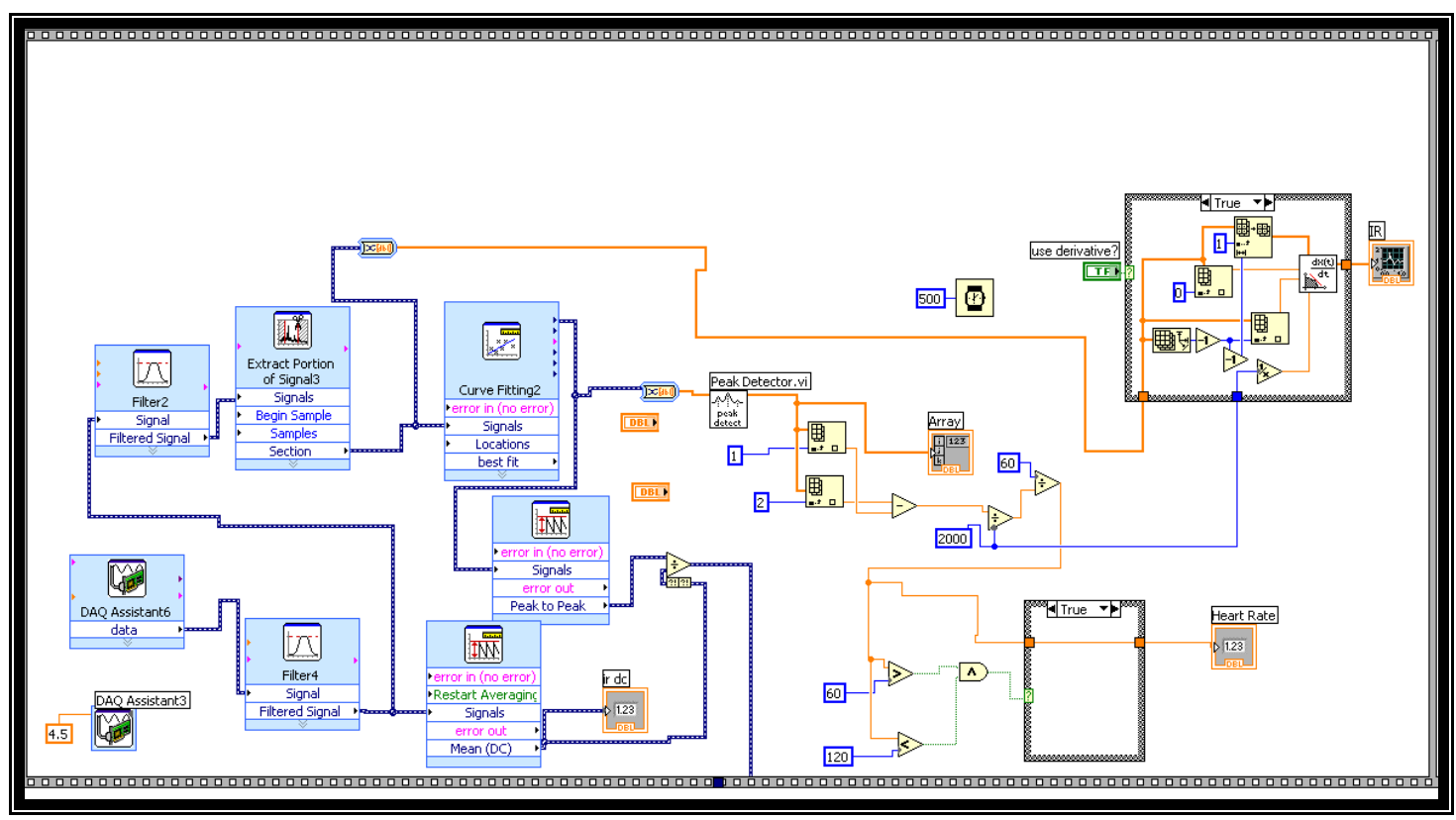

Fig 3(b): VI Block diagram of Pulse Oximeter for turning IR LED ON and OFF 


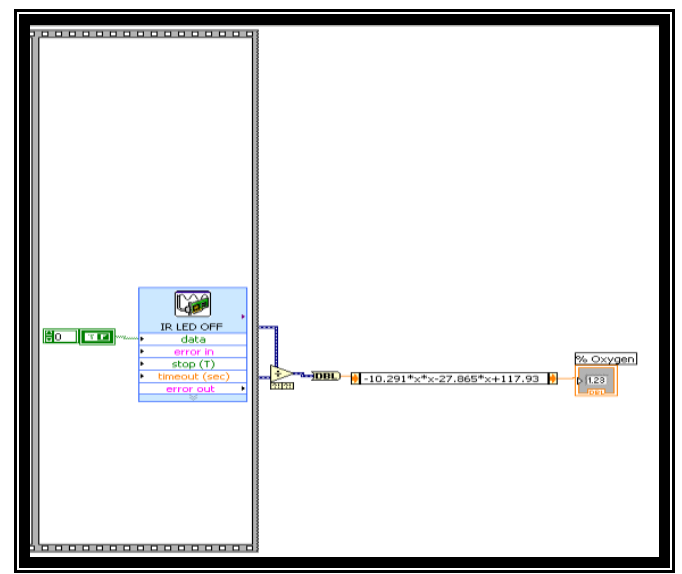

Fig 3(c): VI Block diagram of Pulse Oximeter for calculating percentage of oxygen

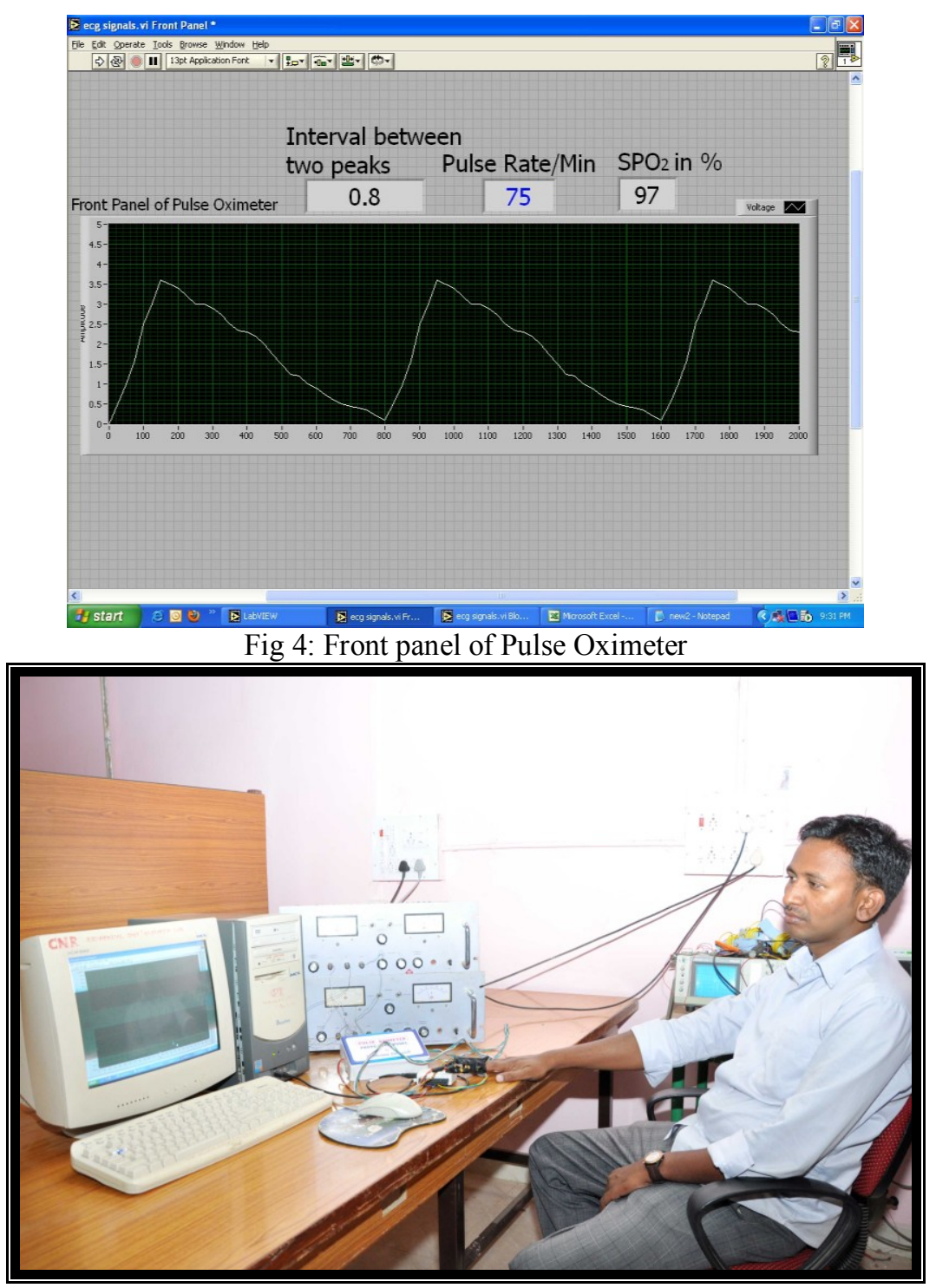

Fig 4.10(c): Measurement of $\mathrm{SPO}_{2}$

\section{Results:}

The measurements which were carried out on the system is in agreement with values measured with standard BPL pulse Oximeter. The measurements were carried out on all age groups. The total measurements calibrated with an accuracy of $98 \%$. The error was not allowed beyond $2 \%$. The sampled and measured data is compared with the standard instrument. As the readings are in good agreement with standard instrument, the 
measurements have been recorded on LabVIEW and displayed on the front panel shown in fig 4. Hence Measurement of $\mathrm{SPO}_{2}$ using LabVIEW is also a part of integrated instrumentation system for patient's monitoring.

\section{Journal Papers:}

\section{REFERENCES}

[1]. Tytler JA, Seeley HF. The Nellcor N-101 pulse oximeter. A clinical evaluation in anaesthesia and intensive care. Anaesthesia 41: 302-305, 1986 .

[2]. Matthes, K., Untersuchungen uber die sauerstoffsattigungen menschlichen arterienblutes, Arc. Exp. Pathol. Parmakol. 179, 689, 1935 .

[3]. Wood, E. and Geraci, J.E., Photoelectric determination of arterial oxygen saturation in man, J. Lab Clin. Med., 34, $387,1949$.

[4]. Tytler JA, Seeley HF. The Nellcor N-101 pulse oximeter. A clinical evaluation in anaesthesia and intensive care. Anesthesia 41: $302-305,1986$.

\section{Books:}

[5]. Merrick, E.B. and T.J. Hayes, continuous, non invasive measurements of arterial blood oxygen level, Hewlett-Packard J., p 2 Oct.1976.

[6]. J Aoyagi, T., Kishi,T., Yamaguchi, K., and Watanabe,S., Improvement of ear piece oximator,in Proc. 13th Japan. Medical Electronics and Biological Engineering Conf., Osaka 90,1974.

[7]. Technical Manual from National Instruments. www.NI.com

[8]. Virtual Instrumentation Using LabVIEW- Jovitha Jerome

\section{Theses:}

[9]. Yun-Thai Li, “ Pulse Oximetry “,Department of Electronic Engineering, University of Surrey, Guildford, GU2 7XH 